OPEN ACCESS

Edited by:

Yanmei $L i$,

Tsinghua University, China

Reviewed by:

Harald Kolmar

Darmstadt University of

Technology, Germany

Francisco Solano,

University of Murcia, Spain

*Correspondence:

Richard Strasser

richard.strasser@boku.ac.at

Specialty section:

This article was submitted to

Chemical Biology,

a section of the journal

Frontiers in Chemistry

Received: 20 January 2020

Accepted: 02 April 2020

Published: 22 April 2020

Citation:

Göritzer K, Goet I, Duric S, Maresch D, Altmann $F$, Obinger $C$ and Strasser $R$ (2020) Efficient N-Glycosylation of the

Heavy Chain Tailpiece Promotes the

Formation of Plant-Produced Dimeric

IgA. Front. Chem. 8:346.

doi: 10.3389/fchem.2020.00346

\section{Efficient $\mathrm{N}$-Glycosylation of the Heavy Chain Tailpiece Promotes the Formation of Plant-Produced Dimeric $\lg \mathrm{A}$}

\author{
Kathrin Göritzer ${ }^{1}$, Iris Goet ${ }^{1}$, Stella Duric ${ }^{1}$, Daniel Maresch ${ }^{2}$, Friedrich Altmann ${ }^{2}$, \\ Christian Obinger $^{2}$ and Richard Strasser ${ }^{1 *}$
}

${ }^{1}$ Department of Applied Genetics and Cell Biology, Institute for Plant Biotechnology and Cell Biology, University of Natural Resources and Life Sciences, Vienna, Austria, ${ }^{2}$ Division of Biochemistry, Department of Chemistry, University of Natural

Resources and Life Sciences, Vienna, Austria

Production of monomeric IgA in mammalian cells and plant expression systems such as Nicotiana benthamiana is well-established and can be achieved by co-expression of the corresponding light and heavy chains. In contrast, the assembly of dimeric IgA requires the additional expression of the joining chain and remains challenging especially in plant-based systems. Here, we examined factors affecting the assembly and expression of HER2 binding dimeric $\lg A 1$ and $\operatorname{lgA} 2 \mathrm{~m}(2)$ variants transiently produced in $N$. benthamiana. While co-expression of the joining chain resulted in efficient formation of dimeric IgAs in HEK293F cells, a mixture of monomeric, dimeric and tetrameric variants was detected in plants. Mass-spectrometric analysis of site-specific glycosylation revealed that the $\mathrm{N}$-glycan profile differed between monomeric and dimeric IgAs in the plant expression system. Co-expression of a single-subunit oligosaccharyltransferase from the protozoan Leishmania major in N. benthamiana increased the $N$-glycosylation occupancy at the C-terminal heavy chain tailpiece and changed the ratio of monomeric to dimeric IgAs. Our data demonstrate that $N$-glycosylation engineering is a suitable strategy to promote the formation of dimeric IgA variants in plants.

Keywords: glyco-engineering, glycosylation, immunoglobulin, monoclonal antibody, recombinant glycoprotein

\section{INTRODUCTION}

Human immunoglobulin A (IgA) is the second most prevalent serum immunoglobulin after IgG and is the predominant antibody class in the external secretions of mucosal surfaces, where it serves as a first line of defense against invading pathogens. The human body expends a considerable amount of energy producing IgA variants thereby exceeding the daily production of all other immunoglobulin classes combined (Woof and Mestecky, 2005). This huge IgA demand highlights the importance of IgA in immune defense processes for which it is equipped with unique structural attributes of its heavy chain and its ability to form monomeric, dimeric and polymeric forms (Figure 1). While monomeric IgA is mainly found in serum and consists of two heavy chains (HC) and two light chains (LC), mucosal IgA is mainly dimeric whereby two IgA monomers are linked together by the incorporation of one joining chain (JC). In the dimeric IgA, the JC is covalently linked by disulfide bonds to the penultimate cysteine residue present in the C-terminal tailpiece 
of the IgA HC. The binding of the JC to the 18 amino acid long HC tailpiece is necessary for the formation of dimeric and polymeric IgA variants in the endoplasmic reticulum (ER) (Atkin et al., 1996; Yoo et al., 1999).

While recombinant IgGs are currently most widely used as therapeutic antibodies to combat infections or diseases, alternative antibody formats are gaining attention as potential biopharmaceuticals due to their specific structural properties and binding to different immune receptors (Loos et al., 2014; Brandsma et al., 2019; Montero-Morales et al., 2019). Polymeric antibody formats have a higher valency of antigen-binding sites which has several advantages compared to monomeric antibodies. Recombinant dimeric IgA against EGFR has been shown to be more effective in tumor cell killing than monomeric IgA or IgG1 due to the recruitment of a distinct repertoire of effector functions (Lohse et al., 2011). Moreover, dimeric IgA can bind to the polymeric immunoglobulin receptor (pIgR) and the pIgR-mediated transcytosis of dimeric IgA enables the access to therapeutic targets within the luminal side of mucosal tissues that are inefficiently targeted by current IgG therapeutics (Olsan et al., 2015). On the other hand, the formation of complexes between antiviral dimeric IgAs and viruses prevents the penetration of mucosal barriers (Ruprecht et al., 2019) and contributes to protection against mucosal virus challenge (Watkins et al., 2013). Together, these studies demonstrate the great potential of recombinant dimeric IgA as therapeutic agent. However, the production of dimeric IgA for clinical studies is technologically challenging due to the complex subunit assembly and extensive glycosylation (Vasilev et al., 2016).

Plants are attractive systems for the manufacturing of recombinant biopharmaceuticals including monoclonal antibodies (Stoger et al., 2014). Plant and mammalian cells share a common machinery for the biosynthesis and processing of $\mathrm{N}$-glycans that is conserved up to the initiation of complextype $N$-glycan formation in the cis/medial-Golgi (Strasser, 2016). On plant produced recombinant glycoproteins, complex and truncated $N$-glycans with $\beta 1,2$-xylose and core $\alpha 1,3$-fucose are frequently found. These non-human residues are potentially immunogenic and numerous strategies have been employed to prevent their attachment to $\mathrm{N}$-glycans (Montero-Morales and Steinkellner, 2018). These efforts resulted in the formation of human-like $\mathrm{N}$-glycan structures on recombinant glycoproteins including different immunoglobulin classes (Strasser et al., 2008; Loos et al., 2014; Göritzer et al., 2017; Montero-Morales et al., 2019) and showed that plants tolerate extensive engineering of their glycan structures. Different plant expression systems have been used to produce monomeric, dimeric or secretory IgA variants (Ma et al., 1995; Karnoup et al., 2005; Juarez et al., 2013; Virdi et al., 2013; Paul et al., 2014; Westerhof et al., 2014, 2015; Dicker et al., 2016; Göritzer et al., 2019).

In a previous study, we have transiently expressed monomeric HER2 binding IgA1 and two IgA2 allotypes in leaves of $N$. benthamiana to examine the site-specific $N$-glycosylation (Göritzer et al., 2017). While we found no differences in glycosylation efficiency on most of the $N$-glycosylation sites compared to human cell-derived IgAs, the $N$-glycosylation site present in the tailpiece of all plant-produced IgAs was only $40-60 \%$ glycosylated. Here, we investigated how efficient dimeric HER2 binding IgAs are produced in the transient $N$. benthamiana expression system and whether the $N$-glycosylation in the tailpiece plays a role in the assembly of multimeric IgA.

\section{MATERIALS AND METHODS Construct Design and Cloning}

All constructs used for the expression of monomeric human HER2 binding $\operatorname{IgA} 1$ and $\operatorname{IgA} 2 \mathrm{~m}(2)$ isotypes in $N$. benthamiana and HEK293F cells have been described in detail recently (Göritzer et al., 2017, 2019). The codon optimized gene for expression of the joining chain (JC) (AK312014.1) in either $N$. benthamiana or HEK293F cells was synthesized by GeneArt (Thermo Fisher Scientific, USA) and was flanked with the signal peptide from barley alpha-amylase (AAA98615) and the restriction sites XhoI/AgeI (for expression in N. benthamiana) or the signal peptide "MELGLSWIFLLAILKGVQC" and the restriction sites BamHI/SalI (for expression in HEK293F). The synthesized DNA fragments were cloned into the binary vector pEAQ-HT (Sainsbury et al., 2009) and the mammalian expression vector gWIZ (Genlantis, San Diego, CA) for expression of dimeric IgA in N. benthamiana and HEK293F, respectively, as described previously (Göritzer et al., 2017, 2019).

For the expression of the marginal zone B and B1-cellspecific protein (MZB1) (Q8WU39) in N. benthamiana the codon-optimized MZB1 coding sequence was synthesized by GeneArt. A construct for the expression of a tagged MZB1 (mRFP-MZB1) was obtained by amplification with the primers “TATATCTAGAGATAGGGCTCCTCTTACTGCTA”“TATAG GATCCTCAAAGTTCCTCTCTGGTAGC," digestion with the

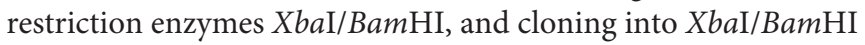
digested p117 (Shin et al., 2017). For the expression of BiP2, the coding sequence was amplified from $A$. thaliana cDNA using the primers "TACTAGTATGGCTCGCTCGTTTGGAGC AAACAGCACT”/"TACTAGTCTAGAGCTCATCGTGAGA CTCATCT" and subcloned using a Zero Blunt TOPO PCR Cloning Kit (Thermo Fisher Scientific, USA). The cloned fragment was excised by SpeI digestion and ligated into XbaI digested expression vector $\mathrm{p} 42$. Vector $\mathrm{p} 42$ is derived from pPT2M (Strasser et al., 2005) and carries the A. thaliana ubiquitin 10 promoter instead of the CaMV35S promoter and the sequence for the attachment of a 3x HA-tag plus the HDEL peptide at the C-terminus of the expressed protein. The CRT2 coding sequence was amplified from $A$. thaliana cDNA using the primers "TATATCTAGAATGGCGAAAATGATTCCTA GCC”/"TATAGGATCCAGCGGTGGCGTCTTTCTCAGAGG." The PCR product was $\mathrm{XbaI} / \mathrm{Bam} \mathrm{HI}$ digested and cloned into the expression vector p59 (Schoberer et al., 2019) to express CRT2 fused to mRFP-HDEL. CNX1 was amplified from $A$. thaliana cDNA using "TATATCTAGAGACGATCAAACGGTT CTGTATG”/"TATAGGATCCCTAATTATCACGTCTCGGTT GCC," XbaI/BamHI digested and cloned into expression vector p110 (same vector as p117 but with a kanamycin resistance gene for selection in plants) to express an mRFP-CNX1 variant. For endoplasmic reticulum resident protein 44 (ERp44) expression, a codon-optimized coding sequence of human 
ERp44 (CAC87611) including the sequence coding for the signal peptide from barley alpha-amylase was synthesized by GeneArt and cloned into the $\mathrm{XbaI} / \mathrm{BamHI}$ sites of $\mathrm{pPT} 2 \mathrm{M}$. The expression construct of the single-subunit oligosaccharyltransferase from Leishmania major (LmSTT3D) has been described previously (Castilho et al., 2018).

\section{Expression and Purification of Dimeric IgA}

For the expression of different recombinant monomeric and dimeric IgA isotypes in 5 to 6 weeks old $N$. benthamiana $\triangle \mathrm{XT} / \mathrm{FT}$ plants, syringe-mediated agro-infiltration was used (Strasser et al., 2008; Göritzer et al., 2017). To obtain dimeric IgA variants, the $\kappa-\mathrm{LC}$ and respective $\alpha-\mathrm{HC}$ were co-infiltrated with the JC with an $\mathrm{OD}_{600}$ of 0.1 or 0.2 . Chaperones were coinfiltrated at an $\mathrm{OD}_{600}$ of 0.05 . To increase the $N$-glycosylation occupancy, IgAs were co-infiltrated with LmSTT3D at an $\mathrm{OD}_{600}$ of 0.1 . After 4 days, infiltrated leaf material was harvested and the clarified crude extract was prepared for IgA purification as described previously (Göritzer et al., 2017). For the transient expression of monomeric and dimeric IgA isotypes in HEK293F cells, cultures were transfected with the $\kappa$-LC, the different $\alpha$ HCs and JC constructs in a 1:1:0 and 1:1:0.5 ratio of $\mu \mathrm{g}$ DNA, respectively, as described (Göritzer et al., 2019). Finally, IgA from clarified $N$. benthamiana $\Delta \mathrm{XT} / \mathrm{FT}$ leaf extract and supernatant of HEK293F cells was purified with IgA CaptureSelect affinity resin (Thermo Fisher Scientific, US), followed by a size-exclusion chromatography step (Göritzer et al., 2017).

\section{SDS-PAGE}

For reducing or non-reducing SDS-PAGE $2.5 \mu \mathrm{g}$ of purified protein were loaded on a $4-15 \%$ Mini-PROTEAN ${ }^{\circledR}$ TGX ${ }^{\mathrm{TM}}$ gel (Bio-Rad laboratories, USA) and detected by Coomassie Brilliant Blue staining.

\section{Size-Exclusion Chromatography Coupled to Multi-Angle Light Scattering (SE-HPLC-MALS)}

To investigate the oligomeric state, conformational integrity and molecular weight of purified IgAs, high performanceliquid-chromatography (HPLC) coupled to a size-exclusion chromatography column (Superdex 200 10/300 GL column, GE Healthcare, USA) combined with multi-angle light scattering were carried out as described previously (Göritzer et al., 2017). HPLC (Shimadzu prominence LC20) was equipped with MALS (WYATT Heleos Dawn8+ QELS; software ASTRA6), refractive index detector (RID-10A, Shimadzu) and a diode array detector (SPD-M20A, Shimadzu). Ratios of monomeric, dimeric and polymeric IgA were determined by peak-integration using LabSolutions Data Analysis (Shimadzu) software.

\section{ELISA}

Purified human HER2 (residues 1-631) was provided by Elisabeth Lobner (University of Natural Resources and Life Sciences, Vienna). For antigen-binding experiments of monomeric, dimeric and polymeric IgA variants ELISA was performed as described recently (Göritzer et al., 2017).

\section{Surface Plasmon Resonance (SPR) Spectroscopy}

Binding experiments of monomeric and dimeric IgA variants to FcaRI were performed with surface plasmon resonance spectroscopy using a Biacore T200 (GE Healthcare Life Sciences, Sweden). Recombinant soluble FcaRI was available from a previous study (Göritzer et al., 2019). All measurements were conducted with a Protein L sensor chip (GE Healthcare Life Sciences, Sweden) as described recently (Göritzer et al., 2019). Binding affinities $\left(K_{\mathrm{D}}\right)$ were calculated with the Biacore T2

\section{A IgA subclasses}

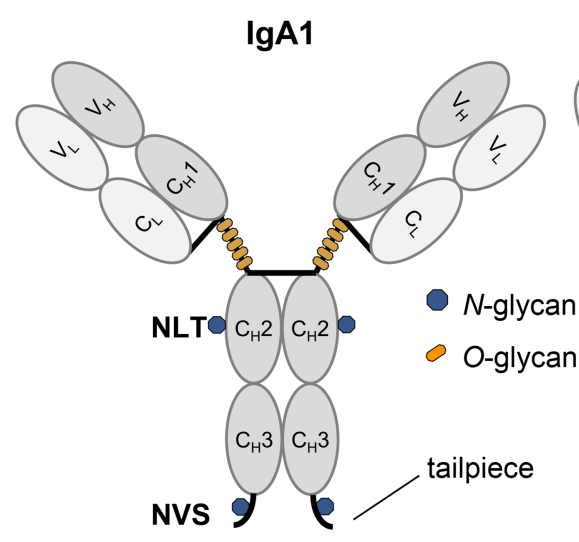

$\lg A 2 m(2)$

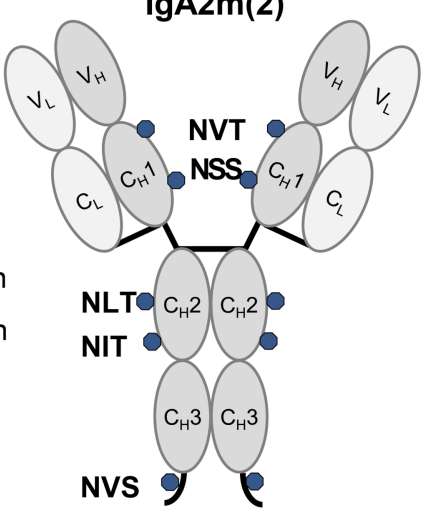

B Multimeric forms of $\lg \mathrm{A}$
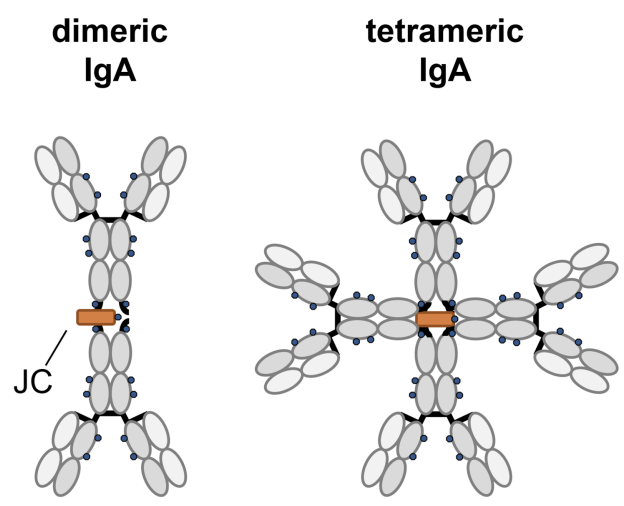

FIGURE 1 | Schematic illustration of structure and glycosylation of IgA variants. (A) Representation of the IgA isotypes IgA1 and IgA2m(2) with the light chain colored in light gray and the heavy chain in dark gray, $\mathrm{N}$-glycans are indicated by blue dots, O-glycans specific for the elongated hinge-region of IgA1 are indicated by orange dots. (B) Schematic presentation [only for $\lg A 2 \mathrm{~m}(2)$ shown] of multimeric states with the joining chain in orange. 


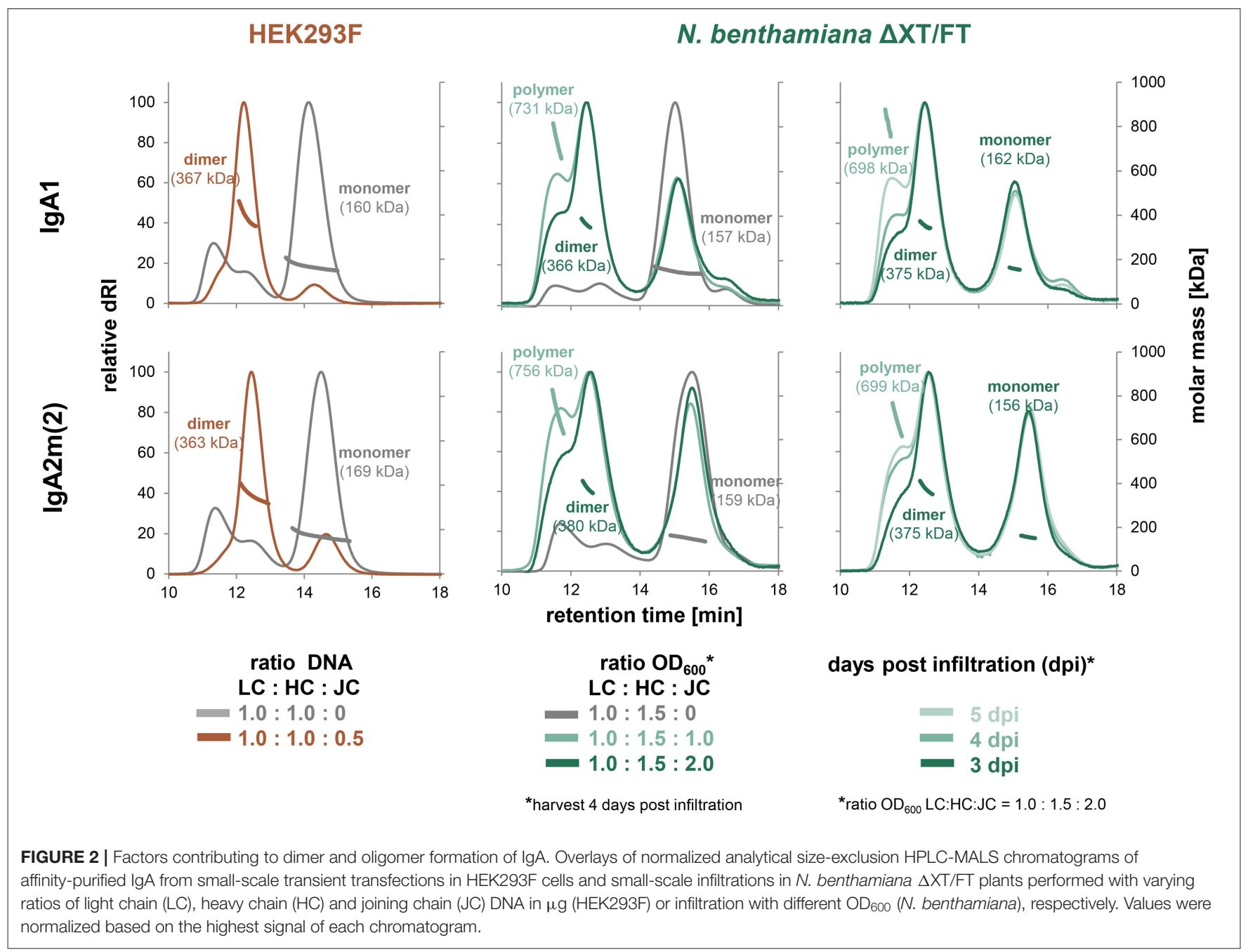

Evaluation software using a 1:1 binding model. All experiments were repeated as three independent kinetic runs.

\section{N-Glycan Analysis}

A total of $20 \mu \mathrm{g}$ purified protein was reduced, S-alkylated and digested with trypsin (Promega, USA). Glycopeptides were then analyzed by capillary reversed-phase chromatography and electron-spray mass spectrometry using a Bruker Maxis 4G QTOF instrument (Göritzer et al., 2017). Site-specific glycosylation occupancy was calculated using the ratio of deamidated to unmodified peptide determined upon $N$-glycan release with PNGase A (Europa Bioproducts).

\section{RESULTS}

\section{Dimeric IgA Variants Are Less Efficiently Formed in $\mathbf{N}$. benthamiana}

To obtain a better understanding of dimeric $\operatorname{IgA}$ assembly, HER2 binding monomeric and dimeric IgA1 and $\operatorname{IgA} 2 \mathrm{~m}(2)$ (Figure 1) were transiently expressed in HEK293F cells and glyco-engineered $N$. benthamiana $\Delta \mathrm{XT} / \mathrm{FT}$ plants. Therefore, the
$\kappa$-LC and respective $\alpha$-HC were co-expressed in the presence and absence of the JC, followed by affinity purification and analysis of the assembly using SE-HPLC coupled to multiangle light scattering (MALS). This allowed the determination of the molecular mass of the proteins in solution and quantification of the relative amounts of the different species using peak integration. Size-exclusion chromatograms showed that relatively pure monomers of $\operatorname{IgA} 1$ and $\operatorname{IgA} 2 \mathrm{~m}(2)$ with a mass of $\sim 160 \mathrm{kDa}$ are produced in the absence of the JC. In both expression systems, only small amounts of IgA with a molecular weight $>160 \mathrm{kDa}$ could be observed (Figure 2). Cotransfection of the JC resulted in almost complete formation of dimeric IgAs with a molecular mass of around $360 \mathrm{kDa}$ in HEK293F cells. By contrast, a mixture of monomeric, dimeric and polymeric species was observed in plants. Thereby, the assembly of dimeric IgA1 appeared to be more efficient than the assembly of dimeric $\operatorname{IgA} 2 \mathrm{~m}(2)$. The formation of polymeric IgA, however, was dependent on the relative amount of JC cotransfected with the $\kappa-\mathrm{LC}$ and $\alpha-\mathrm{HC}$ and the harvesting time after infiltration. Increasing ratios of JC to $\kappa-\mathrm{LC}$ and $\alpha-\mathrm{HC}$ in the infiltration mix resulted in a decreased percentage of 


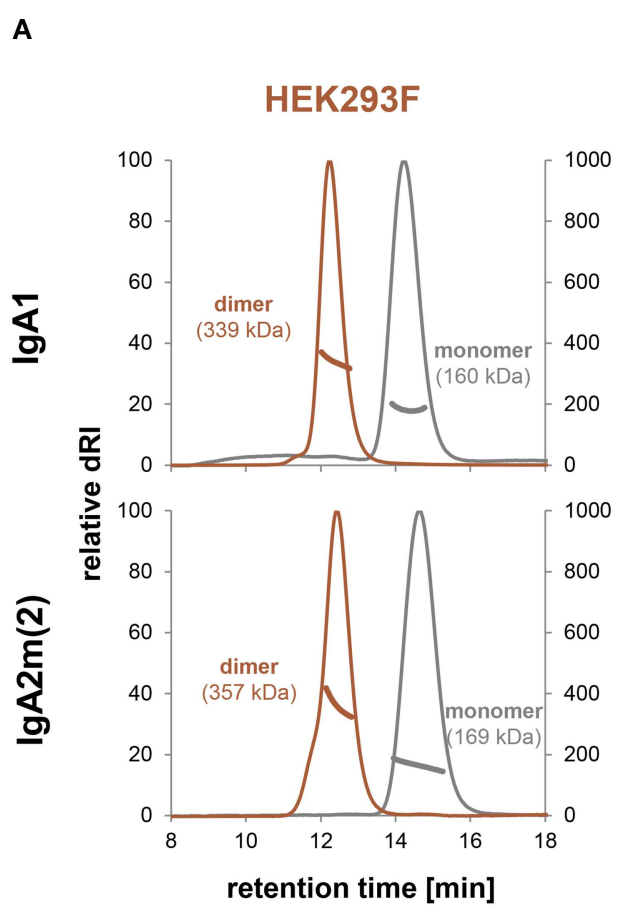

\section{N. benthamiana $\Delta \mathrm{XT} / \mathrm{FT}$}

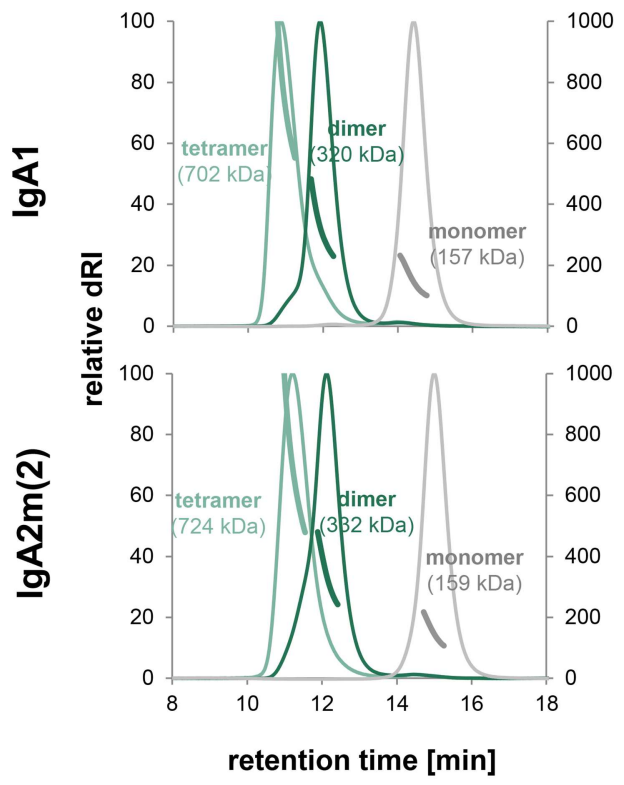

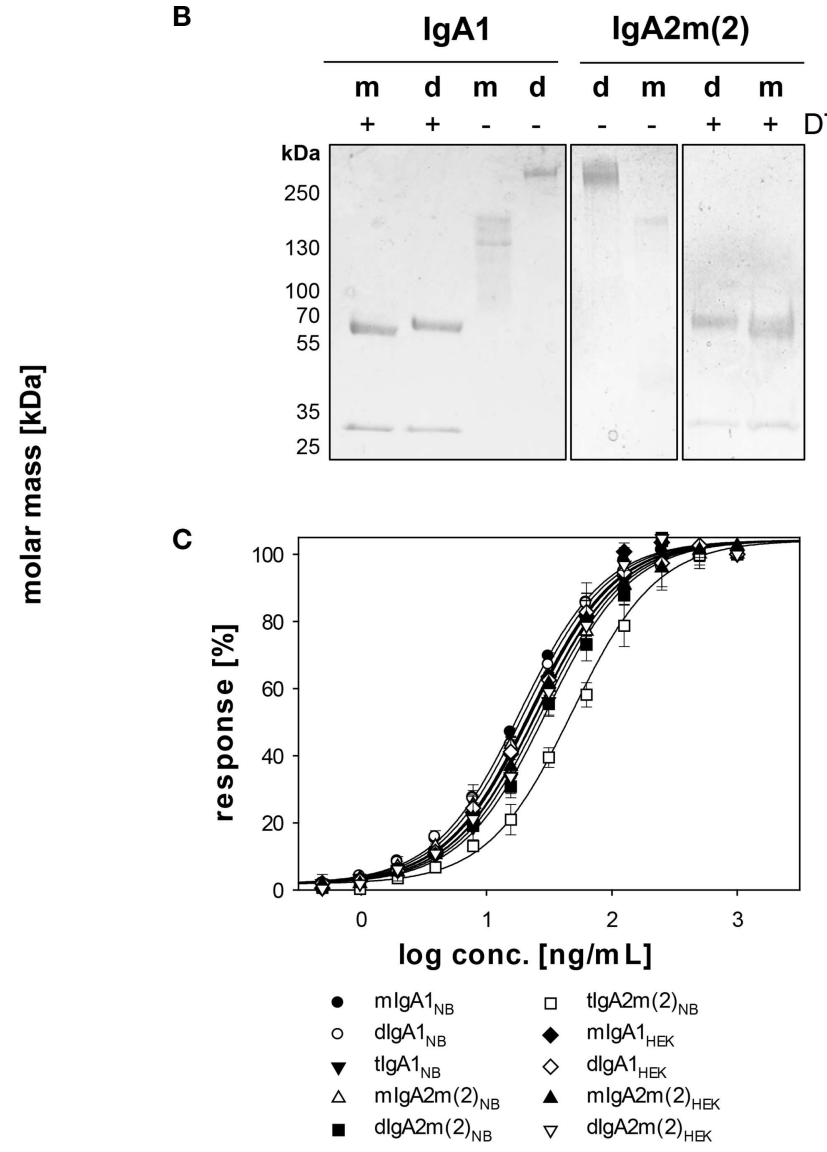

D

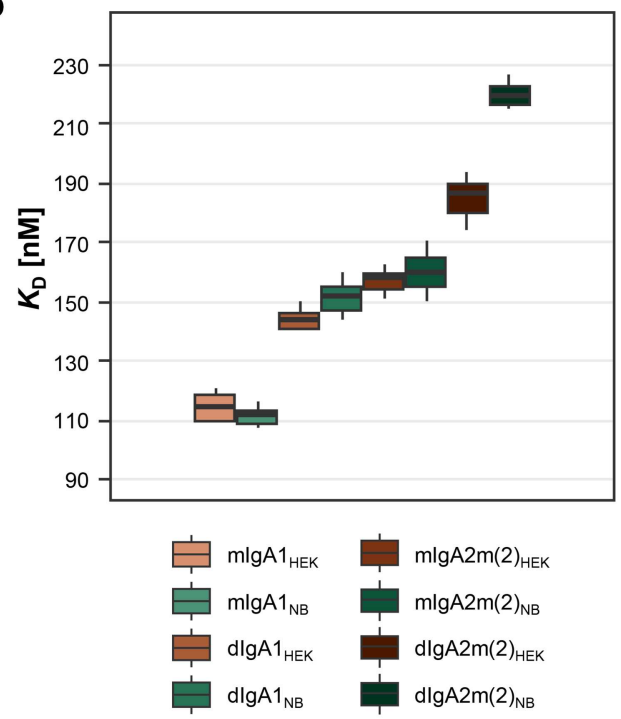

FIGURE 3 | Biophysical and functional characterization of recombinant monomeric, dimeric and tetrameric IgA. (A) Overlay of normalized SE-HPLC-MALS chromatograms of affinity and gel-filtration purified lgA1 and IgA2m(2) monomers, dimers and tetramers produced in HEK293F cells and N. benthamiana $\triangle X T / F T$ plants. (B) SDS-PAGE under reducing (+DTT) and non-reducing conditions of purified monomeric (m) and dimeric (d) IgA1 and IgA2m(2) produced in N. benthamiana $\triangle X T / F T$ plants followed by Coomassie Brilliant Blue staining. (C) Binding of the IgA variants to the antigen HER2. The EC 50 vales were determined as the mean \pm standard deviation from three independent measurements. "m" monomeric, "d" dimeric, "t" tetrameric IgA. (D) Binding affinities of IgA1 and IgA2m(2) monomers and dimers to FcaRI. $K_{D}$ values were obtained by SPR spectroscopy in single-cycle kinetic experiments from three independent measurements. Error bars represent standard deviation. 

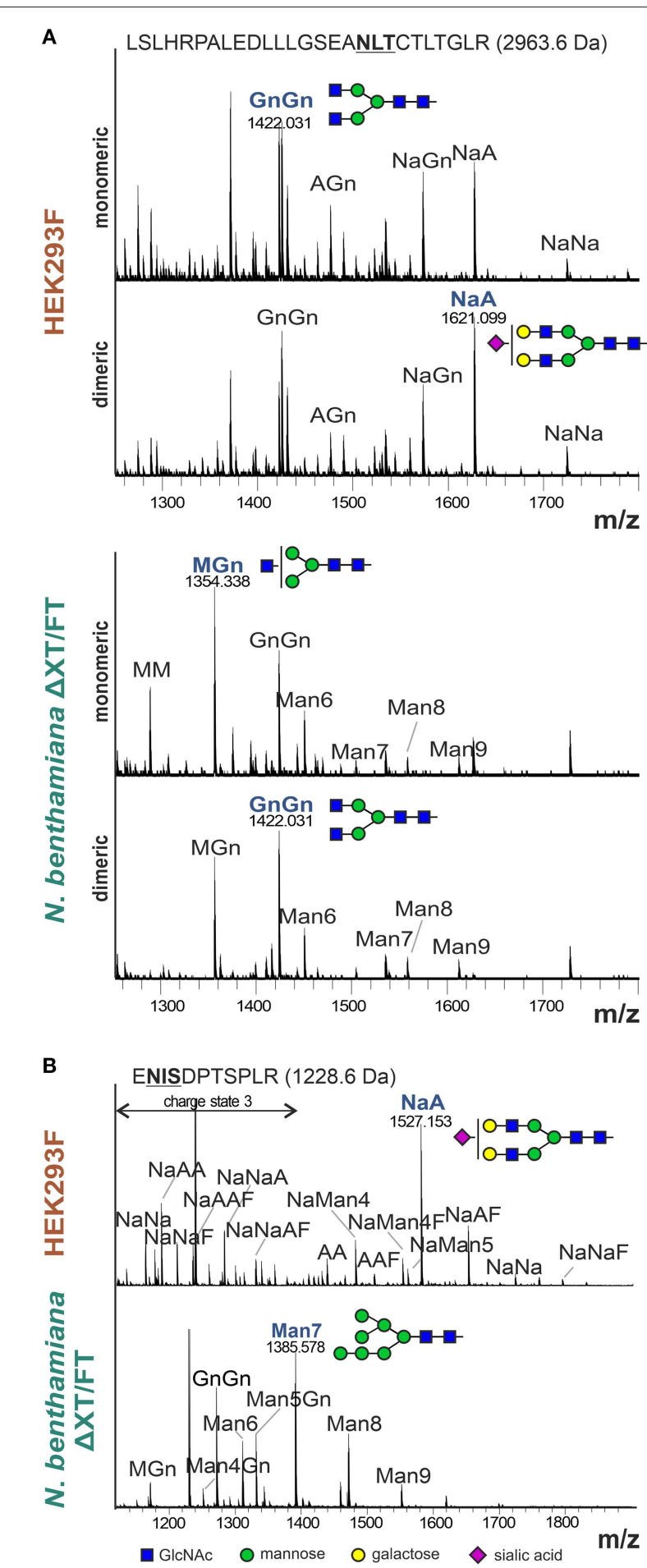

FIGURE 4 | $N$-glycan analysis of the $\alpha-\mathrm{HC}$ and JC from purified monomeric and dimeric IgA1. (A) Representative MS-spectra $\left([\mathrm{M}+3 \mathrm{H}]^{3+}\right)$ of the tryptic glycopeptide containing the $\mathrm{CH} 2$ resident NLT glycosylation site of the $\alpha-\mathrm{HC}$ of HEK293F- and plant-produced IgA1. (B) Representative MS-spectra $\left([\mathrm{M}+2 \mathrm{H}]^{2+}\right.$ and $\left.[\mathrm{M}+3 \mathrm{H}]^{3+}\right)$ of the tryptic glycopeptide containing the single NIS glycosylation site of the HEK293F- and plant-produced dimeric IgA1 joining chain. $\mathrm{N}$-glycans are abbreviated according to the ProGlycAn system (www.proglycan.com). The most abundant glycoform is highlighted in blue and illustrated with cartoons. polymeric IgA. Furthermore, a later harvesting point yielded higher amounts of polymeric IgA (Figure 2 and Figure S1).

\section{Monomeric and Dimeric/Tetrameric IgA Display Similar Antigen and Receptor Binding Affinities}

To determine the biophysical and biochemical properties of different IgA forms we up-scaled the production in both expression systems, followed by separate isolation of monomeric, dimeric and tetrameric species after affinity chromatography using preparative size-exclusion chromatography. The analytical profiles of all purified variants from the two different expression systems gave narrow single and monodisperse peaks (Figure 3A). The masses of these peaks were confirmed by MALS and represent fully assembled monomeric, dimeric and tetrameric IgA (present in plant-derived variants), with masses corresponding well to their theoretical masses. The SDS-PAGE of plant-produced monomeric and dimeric IgA1 and $\operatorname{IgA} 2 \mathrm{~m}(2)$ under reducing conditions confirmed the presence of the $\kappa-\mathrm{LC}$ and $\alpha-\mathrm{HC}$ without the presence of degradation products (Figure 3B). Interestingly, under reducing conditions a small shift in the migration behavior of the $\alpha-\mathrm{HC}$ of dimeric compared to monomeric $\operatorname{IgA} 1$ and $\operatorname{IgA} 2 \mathrm{~m}(2)$ was observed, which could arise from the presence of differentially processed $\mathrm{N}$-glycans on monomeric and dimeric IgA. The JC (15 kDa) of dimeric variants could not be detected on the gel likely due to its low abundance. Under non-reducing conditions purified monomeric and dimeric $\operatorname{IgA} 1$ and $\operatorname{IgA} 2 \mathrm{~m}(2)$ variants showed a predominant band in the range of the expected molecular mass of $160 \mathrm{kDa}$ for each monomeric variant and at $320 \mathrm{kDa}$ for each dimeric variant, representing the assembled forms.

Next, we investigated the functionality of all purified IgAs in terms of binding to the antigen HER2. ELISA experiments were performed and the half-maximal effective concentrations $\left(\mathrm{EC}_{50}\right)$ were determined (Figure 3C). Thereby it could be shown that the antigen binding behavior of monomeric and dimeric IgA1 and $\operatorname{IgA} 2 \mathrm{~m}(2)$ from plant and mammalian hosts is essentially the same and only tetrameric $\operatorname{IgA} 2 \mathrm{~m}(2)$ expressed in N. benthamiana showed slightly decreased binding to the antigen.

The monomeric and dimeric variants of $\operatorname{IgA} 1$ and $\operatorname{IgA} 2 \mathrm{~m}(2)$ were further tested for binding to the Fc $\alpha$-receptor (Fc $\alpha \mathrm{RI})$ using surface plasmon resonance (SPR) spectroscopy (Figure 3D). Therefore, the different IgA variants were immobilized on a Protein L chip in an oriented manner with the Fc-domain pointing toward the solution. Five increasing concentrations of the FcaRI were injected in single-cycle kinetic experiments. The obtained response units suggested a 1:1 binding stoichiometry for monomeric and dimeric IgA variants to the receptor and curves were fitted accordingly. The $K_{\mathrm{D}}$ values around $110 \mathrm{nM}$ and $170 \mathrm{nM}$ obtained for the HEK293F- and plantderived monomeric $\operatorname{IgA} 1$ and $\operatorname{IgA} 2 \mathrm{~m}(2)$ variants, respectively, corresponded to the previously reported values using this setup (Göritzer et al., 2019). A rapid association and dissociation rate was characteristic for the interaction of FcaRI with all IgA variants, whereas a decreased association rate for dimeric IgAs could be observed resulting in a slightly reduced binding 
A

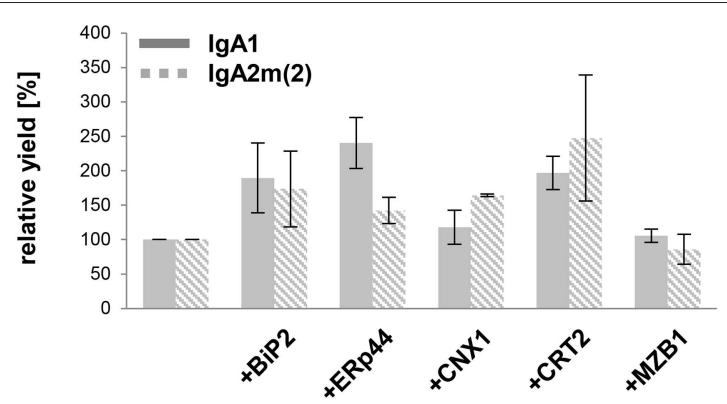

B

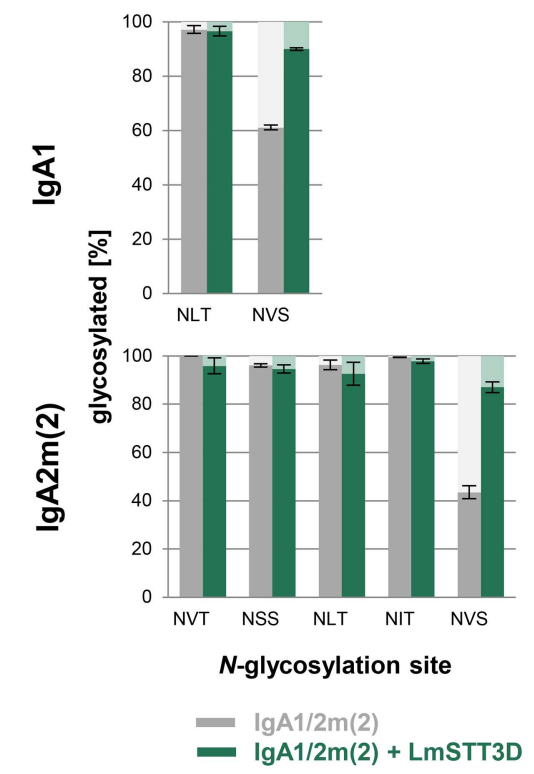

C

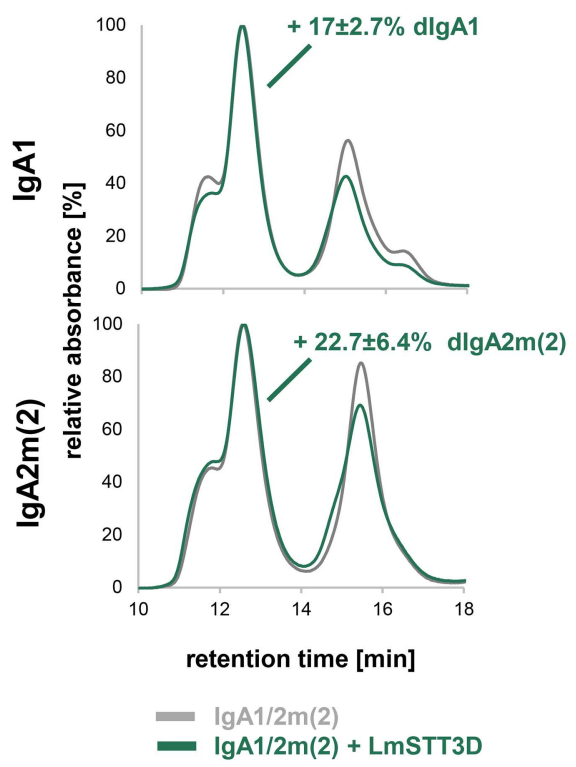

FIGURE 5 | Optimization of dimeric IgA assembly in plants. (A) Relative yields of purified dimeric $\lg A 1$ and $\lg A 2 \mathrm{~m}(2)$ co-infiltrated with different ER-resident proteins in $N$. benthamiana $\triangle \mathrm{XT} / \mathrm{FT}$. The bars indicate the average relative yield and error bars indicate the standard error $(n=4)$. (B) $N$-glycosylation site occupancy of plant-produced dimeric IgA1 and IgA2m(2) either infiltrated alone or co-infiltrated with LmSTT3D. Each value is the mean \pm standard deviation from four independent experiments. (C) Overlay of normalized SE-HPLC chromatograms of affinity-purified dimeric IgA1 and IgA2m(2) with or without co-infiltrated LmSTT3D. The increase of dimeric IgA compared to monomeric and polymeric forms is the mean \pm standard deviation from four independent experiments.

affinity compared to the monomeric IgA variants (Figure 3D and Table S1).

\section{The N-Glycans of Plant-Derived Dimeric IgAs Are Different From Monomeric Variants}

In the reducing SDS-PAGE of monomeric $\operatorname{IgA} 1$ and $\operatorname{IgA} 2 \mathrm{~m}(2)$ produced in $N$. benthamiana the shift in the mobility between monomeric/dimeric forms may arise from differential glycosylation. There are two and five $N$-glycosylation sites in $\operatorname{IgA} 1$ and $\operatorname{IgA} 2 \mathrm{~m}(2)$, respectively (Figure 1). In addition, IgA1 has up to $6 \mathrm{O}$-glycosylation sites in the proline-rich hinge region (Royle et al., 2003; Göritzer et al., 2017). To assess the $\mathrm{N}$-glycosylation status of purified monomeric and dimeric IgA1 and $\operatorname{IgA} 2 \mathrm{~m}(2)$ produced in $N$. benthamiana and HEK293F cells, the purified proteins were digested with trypsin and analyzed by LC-ESI-MS for site-specific $N$-glycosylation and the presence of modifications within the IgA1 hinge region. The $N$-glycans found on plant-produced monomeric IgA1 showed biantennary complex-type structures like GlcNAc$_{1} \mathrm{Man}_{3} \mathrm{GlcNAc}_{2} \quad(\mathrm{MGn} / \mathrm{GnM}), \quad \mathrm{GlcNAc}_{2} \mathrm{Man}_{3} \mathrm{GlcNAc}_{2}$ ( $\mathrm{GnGn}$ ) and the paucimannosidic $\mathrm{Man}_{3} \mathrm{GlcNAc}_{2}(\mathrm{MM})$ as major glycoforms. In addition, small amounts of oligomannosidic $\mathrm{N}$-glycans were detected on some of the sites (Figure 4A and Figures S2, S3). HEK293F-produced monomeric IgA variants have a more diverse profile with different amounts of branched or sialylated complex $N$-glycans. While most of the complex $\mathrm{N}$-glycans are fucosylated Figures S2, S3), the conserved NLT site (Figure 1) lacks core fucose on monomeric as well as on the dimeric HEK293F-derived variants (Figure 4A). This finding is in accordance with the site-specific differences occurring on the monomeric IgA isotypes that have been described (Göritzer et al., 2017). Generally, the $\mathrm{N}$-glycosylation profile of HEK293F-produced dimeric IgAs was similar to monomeric IgAs. For plant-produced dimeric 
IgAs a clear shift of paucimannosidic (MM structures) toward fully processed complex $\mathrm{N}$-glycans (GnGn structures) was observed for the NLT site of dimeric IgA1 (Figure 4A), as well as for the NVT, NSS, and NIT sites of dimeric $\operatorname{IgA2m}(2)$ (Figure S3). By contrast, $O$-glycans present in the IgA1 hinge region appeared similar in monomeric and dimeric variants (Figure S4).

Furthermore, we were able to detect the single glycopeptide corresponding to the JC of HEK293F- and plant-produced dimeric IgA variants (Figure 4B and Figure S5). The $N$-glycan profile of the single site in the JC of HEK293F-produced $\operatorname{IgA} 1$ and $\operatorname{IgA} 2 \mathrm{~m}(2)$ showed a high heterogeneity with high levels of branching and incomplete sialylation and some peaks corresponding to core-fucosylated and hybrid $N$-glycans. In plant-produced JC derived from purified dimeric IgA1 and $\operatorname{IgA} 2 \mathrm{~m}(2)$ variants, the $N$-glycans were more homogenous with the GnGn-type complex $N$-glycan and oligomannosidic structures as major glycoforms and low levels of unglycosylated JC. The presence of oligomannosidic $N$-glycans suggests incomplete processing of the JC $\mathrm{N}$-glycans in the Golgi of plants.

\section{Co-expression of ER-Resident Proteins Increased the Overall Yield, but Did Not Improve Dimeric IgA Formation in $\mathbf{N}$. benthamiana}

Despite the JC co-expression, plants were less efficient in assembly of dimeric IgAs compared to HEK293F cells with still large amounts of monomeric IgA present. Therefore, we investigated whether this limitation can be overcome by co-expression of different ER-resident proteins including the chaperone BiP, which is known to play a role for the antibody assembly (Haas and Wabl, 1983), the protein disulfide isomerase ERp44 and the lectins calnexin (CNX) and calreticulin (CRT). Human ERp44 binds to the tailpiece of the IgM HC, which is quite similar to the IgA tailpiece and promotes IgM polymerization in the ER of mammalian cells (Cortini and Sitia, 2010). The lectins CNX and CRT bind to immature $N$-glycans and promote the folding of glycosylated proteins (Hammond et al., 1994). In addition, we co-expressed the human marginal zone B and B-1 cell-specific protein MZB1, which was recently shown to promote JC binding and dimeric IgA assembly in mammalian cells (Xiong et al., 2019). The $\kappa-L C, \alpha-H C$, and JC were co-infiltrated with either Arabidopsis BiP2, CRT2mRFP, mRFP-CNX1, human ERp44, or mRFP-MZB1. None of these ER-resident proteins increased the relative amount of dimeric to monomeric IgA (Figure S6). However, upon using BiP2, CRT2, CNX1, and ERp44 higher yields could be achieved with up to 2-fold increase of purified IgA per gram fresh weight (Figure 5A).

\section{Increased $\mathbf{N}$-Glycosylation of the Tailpiece Promotes Dimeric IgA Formation in $\mathbf{N}$. benthamiana}

Previous studies in mammals have indicated an important role of the tailpiece $\mathrm{N}$-glycan for JC incorporation (Atkin et al., 1996; Sørensen et al., 2000). In plant-produced IgAs, the tailpiece is incompletely glycosylated which might contribute to inefficient dimeric IgA formation (Westerhof et al., 2015; Göritzer et al., 2017; Castilho et al., 2018). Underglycosylation was even more pronounced in the $\operatorname{IgA} 2 \mathrm{~m}(2)$ isotype which also exhibited higher amounts of non-assembled monomeric IgA when co-infiltrated with the JC. In a previous study we have shown that it is possible to overcome the reduced glycosylation efficiency by co-expression of LmSTT3D, a single subunit oligosaccharyltransferase from the protozoan Leishmania major (Castilho et al., 2018). Using this approach the occupancy of the tailpiece $N$-glycosylation site of dimeric $\operatorname{IgA} 1$ and $\operatorname{IgA} 2 \mathrm{~m}(2)$ increased from 61.1 $\pm 0.9 \%$ to $90.0 \pm 0.4$ and $43.5 \pm 2.7 \%$ to $87 \pm 2.2 \%$, respectively (Figure 5B). However, the almost complete $\mathrm{N}$ glycosylation in the tailpiece did not fully compensate for the reduced dimeric IgA formation compared to HEK293Fproduced IgA variants. The LmSTT3D co-expression led to an $17 \pm 2.7 \%$ increase of dimeric to monomeric $\operatorname{IgA}$ for $\operatorname{IgA} 1$ and an increase of $22.7 \pm 6.4 \%$ for $\operatorname{IgA} 2 \mathrm{~m}(2)$ (Figure 5C). The assembly was also not further improved when, MZB1 and LmSTT3D were co-expressed together with IgA variants (Figure S7) suggesting the involvement of additional factors for dimeric IgA formation that are missing in plants.

\section{DISCUSSION}

In this study we examined factors that affect the dimeric IgA formation in $N$. benthamiana which is currently one of the most widely used plant for recombinant protein expression and glyco-engineering (Bally et al., 2018; MonteroMorales and Steinkellner, 2018). IgAs are heavily glycosylated and distinct glycoforms contribute to the overall thermal stability of IgAs (Göritzer et al., 2017), the in vivo halflife (Rouwendal et al., 2016) and effector functions (Steffen et al., 2020). Moreover, not only is the $N$ - as well as $O$ glycan composition different between plant- and mammalian cell-derived IgAs, but also the degree of glycosylation in the single tailpiece $N$-glycosylation site (Göritzer et al., 2017; Castilho et al., 2018). This underglycosylation at the C-terminal end of the $\alpha$-HC may be caused by unknown differences in the oligosaccharyltransferase function between mammals and plants (Strasser, 2016; Shrimal and Gilmore, 2019). Mammals contain two distinct OST complexes and the STT3B complex post-translationally glycosylates acceptor sites at extreme Cterminal regions (Ruiz-Canada et al., 2009; Shrimal et al., 2013). Plants have also two distinct STT3 subunits, but their role in co- and post-translational $\mathrm{N}$-glycosylation is unknown (Koiwa et al., 2003). Previously it was suggested that partial $N$-glycosylation of the $\alpha$-HC tailpiece and/or the JC impairs secretory IgA assembly in plants (Westerhof et al., 2015). Our data demonstrate that the $\mathrm{N}$-glycosylation in the tailpiece is critical for the formation of dimeric IgAs. Hence, engineering of the plant oligosaccharyltransferase complex toward a more mammalian-like function is a strategy to improve the production 
of dimeric IgAs. While the site-specific $N$-glycosylation of plantproduced $\alpha-\mathrm{HC}$ and the secretory component were shown previously (Dicker et al., 2016; Göritzer et al., 2017), we could determine for the first time the $\mathrm{N}$-glycan profile of a plantproduced JC incorporated into dimeric IgA. In contrast to the $\alpha$-HC tailpiece, an almost complete occupancy was found at the single JC site suggesting that the JC $N$-glycosylation does not contribute to the reduced amounts of dimeric IgAs in plants.

Previously it was revealed that the JC incorporation is the limiting factor for secretory IgA formation (Westerhof et al., 2015). This is consistent with our findings, where we did not observe an increase of dimeric IgA when the amount of infiltrated JC was varied. Along with $N$-glycosylation, our data indicate that there are other factors contributing to the $\operatorname{IgA}$ dimerization. When we tested the effect of plant and mammalian ER-resident proteins involved in protein folding and assembly, we did not observe improved dimeric IgA formation. However, the overall yield of the produced IgAs was increased. This finding suggests that the plant proteins $\mathrm{BiP}, \mathrm{CNX}$, or CRT are not specifically involved in dimeric IgA formation, but in the assembly or stabilization of the $\operatorname{IgA} \alpha-\mathrm{HC}$ and $\kappa-\mathrm{LC}$ resulting in higher amounts of monomeric and dimeric IgA. Human ERp44 expression clearly increased the yield, but had no effect on the IgA polymerization suggesting that it is not required for this process in plants. Despite being expressed and correctly retained in the ER in plants, tagged human MZB1 neither affected the yield nor improved the dimeric IgA formation. The precise mechanism for the MZB1-mediated incorporation of the JC into dimeric IgA is currently unknown (Xiong et al., 2019). There is no homolog of MZB1 present in plants and it is possible that the protein is not functional in N. benthamiana because a potential interaction partner is missing. MZB1 is a member of a large ER-chaperone complex that includes $\mathrm{BiP}$ as well as other folding assistants (Shimizu et al., 2009). Expression of MZB1 variants or the combination of MZB1 with ER-resident mammalian chaperones could be tested in the future to increase the assembly and JC incorporation. In this regard, the use of multi-cassette vectors carrying the $\alpha-\mathrm{HC}, \mathrm{k}-\mathrm{LC}$, JC as well as potential chaperones on the same vector could overcome unbalanced expression in cells (Westerhof et al., 2015) and further boost the dimeric IgA formation.

In previous studies it was discovered that plant-produced IgAs are poorly secreted and the tailpiece harbors a sequence motif for sorting to the vacuole (Hadlington et al., 2003; Paul et al., 2014; Westerhof et al., 2014). We analyzed the amount of secreted IgAs in the presence or absence of co-expressed JC and could not detect an appreciable difference in secretion (Figure S8). The majority of monomeric and dimeric IgA remained inside the cells, while the IgA in the apoplast was degraded. The absence of major degradation products for the IgA variants inside the cells suggests that they are not targeted to the vacuole. Further studies are required to unravel the subcellular compartment where the majority of the monomeric and dimeric IgAs accumulate.

We were interested to examine whether the $N$-glycan composition is different for monomeric or dimeric IgA variants as reported for monomeric and polymeric serum IgA (Oortwijn et al., 2006). On several $N$-glycosylation sites we observed distinct changes in the $\mathrm{N}$-glycan composition with a decrease of truncated $\mathrm{N}$-glycans on plant-produced dimeric IgAs. These structures are likely generated in a post-Golgi compartment by $\beta$-hexosaminidases (Shin et al., 2017) and differences between monomeric and dimeric IgAs can be explained by changes in the accessibility of the $N$-glycans due to the dimer formation and incorporation of the JC. Whether these changes in $\mathrm{N}$ glycan composition cause differences in binding to specific receptors needs to be tested in the future. Previously, we have shown that monomeric IgAs with different $N$-glycans display comparable binding affinities to FcaRI (Göritzer et al., 2019). Here, we performed a kinetic analysis of the dimeric IgA variants and observed similar affinities and kinetics for monomeric IgA. The $N$-glycan composition of dimeric IgA likely does not contribute Fc $\alpha$ RI binding and the JC incorporation does not cause steric hindrance as shown for the secretory component (Vidarsson et al., 2001). Moreover, the deduced 1:1 binding stoichiometry for dimeric IgA variants to the receptor is consistent with models proposing that one dimeric IgA binds only two Fc $\alpha$ RI, although one dimeric IgA has four binding sites (Bonner et al., 2008; Breedveld and van Egmond, 2019).

In conclusion, we show that functional dimeric IgA binding to the antigen as well as to the most relevant $\operatorname{IgA}$ receptor can be produced by transient expression in $N$. benthamiana. The inefficient formation of dimeric IgAs is partly caused by underglycosylation of the $N$-glycosylation in the tailpiece as well as by other factors that need to be uncovered to make plants a suitable expression system for this important class of monoclonal antibodies.

\section{DATA AVAILABILITY STATEMENT}

All datasets generated for this study are included in the article/Supplementary Material.

\section{AUTHOR CONTRIBUTIONS}

KG, IG, SD, and DM conducted the experiments. KG, IG, DM, and $\mathrm{RS}$ analyzed the results. KG, FA, CO, and RS conceived and supervised the experiments. KG and RS wrote the paper. All authors have made a substantial and intellectual contribution to the work and approved it for publication.

\section{FUNDING}

This work was supported by the Austrian Science Fund (FWF) [Doctoral Program BioToP-410 Biomolecular Technology of Proteins (W1224)] and by the FWF Project P31920-B32.

\section{ACKNOWLEDGMENTS}

We thank Professor George Lomonosoff (John Innes Centre, Norwich, UK) and Plant Bioscience Limited (PBL) (Norwich, UK) for supplying the pEAQ-HT expression vector. We thank 
Andreas Loos, Yun-ji Shin, and Christiane Veit (Department of Applied Genetics and Cell Biology, University of Natural Resources and Life Sciences) for assisting in cloning of expression vectors for the ER-chaperones and the BOKU Core Facility Biomolecular \& Cellular Analysis for help with SPR analysis.

\section{REFERENCES}

Atkin, J. D., Pleass, R. J., Owens, R. J., and Woof, J. M. (1996). Mutagenesis of the human IgA1 heavy chain tailpiece that prevents dimer assembly. J. Immunol. $157,156-159$.

Bally, J., Jung, H., Mortimer, C., Naim, F., Philips, J. G., Hellens, R., et al. (2018). The rise and rise of Nicotiana benthamiana: a plant for all reasons. Annu. Rev. Phytopathol. 56, 405-426. doi: 10.1146/annurev-phyto-080417-050141

Bonner, A., Furtado, P. B., Almogren, A., Kerr, M. A., and Perkins, S. J. (2008). Implications of the near-planar solution structure of human myeloma dimeric IgA1 for mucosal immunity and IgA nephropathy. J. Immunol. 180, 1008-1018. doi: 10.4049/jimmunol.180.2.1008

Brandsma, A. M., Bondza, S., Evers, M., Koutstaal, R., Nederend, M., Jansen, J. H. M., et al. (2019). Potent Fc receptor signaling by IgA leads to superior killing of cancer cells by neutrophils compared to IgG. Front. Immunol. 10:704. doi: 10.3389/fimmu.2019.00704

Breedveld, A., and van Egmond, M. (2019). IgA and Fc $\alpha$ RI: pathological roles and therapeutic opportunities. Front. Immunol. 10:553. doi: 10.3389/fimmu.2019.00553

Castilho, A., Beihammer, G., Pfeiffer, C., Göritzer, K., Montero-Morales, L., Vavra, U., et al. (2018). An oligosaccharyltransferase from Leishmania major increases the N-glycan occupancy on recombinant glycoproteins produced in Nicotiana benthamiana. Plant Biotechnol. J. 16, 1700-1709. doi: 10.1111/pbi.12906

Cortini, M., and Sitia, R. (2010). ERp44 and ERGIC-53 synergize in coupling efficiency and fidelity of IgM polymerization and secretion. Traffic 11, 651-659. doi: 10.1111/j.1600-0854.2010.01043.x

Dicker, M., Tschofen, M., Maresch, D., König, J., Juarez, P., Orzaez, D., et al. (2016). Transient glyco-engineering to produce recombinant IgAl with defined $\mathrm{N}$ - and O-glycans in plants. Front Plant Sci. 7:18. doi: 10.3389/fpls.2016.00018

Göritzer, K., Maresch, D., Altmann, F., Obinger, C., and Strasser, R. (2017). Exploring site-specific N-glycosylation of HEK293 and plantproduced human IgA isotypes. J. Proteome Res. 16, 2560-2570. doi: 10.1021/acs.jproteome.7b00121

Göritzer, K., Turupcu, A., Maresch, D., Novak, J., Altmann, F., Oostenbrink, C., et al. (2019). Distinct Fc $\alpha$ receptor $\mathrm{N}$-glycans modulate the binding affinity to immunoglobulin A (IgA) antibodies. J. Biol. Chem. 294, 13995-14008. doi: 10.1074/jbc.RA119.009954

Haas, I. G., and Wabl, M. (1983). Immunoglobulin heavy chain binding protein. Nature 306, 387-389. doi: 10.1038/306387a0

Hadlington, J. L., Santoro, A., Nuttall, J., Denecke, J., Ma, J. K., Vitale, A., et al. (2003). The C-terminal extension of a hybrid immunoglobulin A/G heavy chain is responsible for its Golgi-mediated sorting to the vacuole. Mol. Biol. Cell 14, 2592-2602. doi: 10.1091/mbc.e02-11-0771

Hammond, C., Braakman, I., and Helenius, A. (1994). Role of N-linked oligosaccharide recognition, glucose trimming, and calnexin in glycoprotein folding and quality control. Proc. Natl. Acad. Sci. U.S.A. 91, 913-917. doi: 10.1073/pnas.91.3.913

Juarez, P., Huet-Trujillo, E., Sarrion-Perdigones, A., Falconi, E. E., Granell, A., and Orzaez, D. (2013). Combinatorial analysis of secretory immunoglobulin A (sIgA) expression in plants. Int. J. Mol. Sci. 14, 6205-6222. doi: 10.3390/ijms14036205

Karnoup, A. S., Turkelson, V., and Anderson, W. H. (2005). O-linked glycosylation in maize-expressed human IgA1. Glycobiology 15, 965-981. doi: 10.1093/glycob/cwi077

Koiwa, H., Li, F., McCully, M., Mendoza, I., Koizumi, N., Manabe, Y., et al. (2003). The STT3a subunit isoform of the Arabidopsis oligosaccharyltransferase controls adaptive responses to salt/osmotic stress. Plant Cell 15, 2273-2284. doi: $10.1105 /$ tpc. 013862

\section{SUPPLEMENTARY MATERIAL}

The Supplementary Material for this article can be found online at: https://www.frontiersin.org/articles/10.3389/fchem. 2020.00346/full\#supplementary-material

Lohse, S., Derer, S., Beyer, T., Klausz, K., Peipp, M., Leusen, J. H., et al. (2011) Recombinant dimeric IgA antibodies against the epidermal growth factor receptor mediate effective tumor cell killing. J. Immunol. 186, 3770-3778. doi: 10.4049/jimmunol.1003082

Loos, A., Gruber, C., Altmann, F., Mehofer, U., Hensel, F., Grandits, M. et al. (2014). Expression and glycoengineering of functionally active heteromultimeric IgM in plants. Proc. Natl. Acad. Sci. U.S.A. 111, 6263-6268. doi: 10.1073/pnas.1320544111

Ma, J. K., Hiatt, A., Hein, M., Vine, N. D., Wang, F., Stabila, P., et al. (1995). Generation and assembly of secretory antibodies in plants. Science 268, 716-719. doi: $10.1126 /$ science.7732380

Montero-Morales, L., Maresch, D., Crescioli, S., Castilho, A., Ilieva, K. M., Mele, $\mathrm{S}$., et al. (2019). Glycan engineering and functional activities of $\operatorname{IgE}$ antibodies. Front. Bioeng. Biotechnol. 7:242. doi: 10.3389/fbioe.2019.00242

Montero-Morales, L., and Steinkellner, H. (2018). Advanced plant-based glycan engineering. Front. Bioeng. Biotechnol. 6:81. doi: 10.3389/fbioe.2018.00081

Olsan, E. E., Matsushita, T., Rezaei, M., and Weimbs, T. (2015). Exploitation of the polymeric immunoglobulin receptor for antibody targeting to renal cyst lumens in polycystic kidney disease. J. Biol. Chem. 290, 15679-5686. doi: 10.1074/jbc.M114.607929

Oortwijn, B. D., Roos, A., Royle, L., van Gijlswijk-Janssen, D. J., FaberKrol, M. C., Eijgenraam, J. W., et al. (2006). Differential glycosylation of polymeric and monomeric IgA: a possible role in glomerular inflammation in IgA nephropathy. J. Am. Soc. Nephrol. 17, 3529-3539. doi: 10.1681/ASN.2006040388

Paul, M., Reljic, R., Klein, K., Drake, P. M., van Dolleweerd, C., Pabst, M., et al. (2014). Characterization of a plant-produced recombinant human secretory IgA with broad neutralizing activity against HIV. MAbs 6, 1585-1597. doi: 10.4161/mabs.36336

Rouwendal, G. J., van der Lee, M. M., Meyer, S., Reiding, K. R., Schouten, J., de Roo, G., et al. (2016). A comparison of anti-HER2 IgA and IgG1 in vivo efficacy is facilitated by high N-glycan sialylation of the IgA. MAbs 8, 74-86. doi: 10.1080/19420862.2015.1102812

Royle, L., Roos, A., Harvey, D. J., Wormald, M. R., van Gijlswijk-Janssen, D., Redwan, E.-R., et al. (2003). Secretory IgA N- and O-glycans provide a link between the innate and adaptive immune systems. J. Biol. Chem. 278, 20140-20153. doi: 10.1074/jbc.M301436200

Ruiz-Canada, C., Kelleher, D. J., and Gilmore, R. (2009). Cotranslational and posttranslational N-glycosylation of polypeptides by distinct mammalian OST isoforms. Cell 136, 272-283. doi: 10.1016/j.cell.2008. 11.047

Ruprecht, R. M., Marasini, B., and Thippeshappa, R. (2019). Mucosal antibodies: defending epithelial barriers against HIV-1 invasion. Vaccines 7:E194. doi: 10.3390/vaccines7040194

Sainsbury, F., Thuenemann, E. C., and Lomonossoff, G. P. (2009). pEAQ versatile expression vectors for easy and quick transient expression of heterologous proteins in plants. Plant Biotechnol. J. 7, 682-693. doi: 10.1111/j.1467-7652.2009.00434.x

Schoberer, J., König, J., Veit, C., Vavra, U., Liebminger, E., Botchway, S. W., et al. (2019). A signal motif retains Arabidopsis ER- $\alpha$-mannosidase I in the cis-Golgi and prevents enhanced glycoprotein ERAD. Nat. Commun. 10:3701. doi: 10.1038/s41467-019-11686-9

Shimizu, Y., Meunier, L., and Hendershot, L. M. (2009). pERp1 is significantly upregulated during plasma cell differentiation and contributes to the oxidative folding of immunoglobulin. Proc. Natl. Acad. Sci. U.S.A. 106, 17013-17018. doi: 10.1073/pnas.0811591106

Shin, Y. J., Castilho, A., Dicker, M., Sádio, F., Vavra, U., Grünwald-Gruber, C., et al. (2017). Reduced paucimannosidic N-glycan formation by suppression of 
a specific $\beta$-hexosaminidase from Nicotiana benthamiana. Plant Biotechnol. J. 15, 197-206. doi: 10.1111/pbi.12602

Shrimal, S., and Gilmore, R. (2019). Oligosaccharyltransferase structures provide novel insight into the mechanism of asparagine-linked glycosylation in prokaryotic and eukaryotic cells. Glycobiology 29, 288-297. doi: 10.1093/glycob/cwy093

Shrimal, S., Trueman, S. F., and Gilmore, R. (2013). Extreme C-terminal sites are posttranslocationally glycosylated by the STT3B isoform of the OST. J. Cell. Biol. 201, 81-95. doi: 10.1083/jcb.201301031

Sørensen, V., Rasmussen, I. B., Sundvold, V., Michaelsen, T. E., and Sandlie, I. (2000). Structural requirements for incorporation of J chain into human IgM and IgA. Int. Immunol. 12, 19-27. doi: 10.1093/intimm/12.1.19

Steffen, U., Koeleman, C. A., Sokolova, M. V., Bang, H., Kleyer, A., Rech, J., et al. (2020). IgA subclasses have different effector functions associated with distinct glycosylation profiles. Nat. Commun. 11:120. doi: 10.1038/s41467-019-13992-8

Stoger, E., Fischer, R., Moloney, M., and Ma, J. K. (2014). Plant molecular pharming for the treatment of chronic and infectious diseases. Annu. Rev. Plant Biol. 65, 743-768. doi: 10.1146/annurev-arplant-050213-035850

Strasser, R. (2016). Plant protein glycosylation. Glycobiology 26, 926-939. doi: $10.1093 /$ glycob/cww023

Strasser, R., Stadlmann, J., Schähs, M., Stiegler, G., Quendler, H., Mach, L., et al. (2008). Generation of glyco-engineered Nicotiana benthamiana for the production of monoclonal antibodies with a homogeneous human-like N-glycan structure. Plant Biotechnol. J. 6, 392-402. doi: 10.1111/j.1467-7652.2008.00330.x

Strasser, R., Stadlmann, J., Svoboda, B., Altmann, F., Glössl, J., and Mach, L. (2005). Molecular basis of $\mathrm{N}$-acetylglucosaminyltransferase I deficiency in Arabidopsis thaliana plants lacking complex N-glycans. Biochem. J. 387, 385-391. doi: 10.1042/BJ20041686

Vasilev, N., Smales, C. M., Schillberg, S., Fischer, R., and Schiermeyer, A. (2016). Developments in the production of mucosal antibodies in plants. Biotechnol. Adv. 34, 77-87. doi: 10.1016/j.biotechadv.2015.11.002

Vidarsson, G., van Der Pol, W. L., van Den Elsen, J. M., Vilé, H., Jansen, M., Duijs, J., et al. (2001). Activity of human IgG and IgA subclasses in immune defense against Neisseria meningitidis serogroup B. J. Immunol. 166, 6250-6256. doi: 10.4049/jimmunol.166.10.6250

Virdi, V., Coddens, A., De Buck, S., Millet, S., Goddeeris, B. M., Cox, E., et al. (2013). Orally fed seeds producing designer IgAs protect weaned piglets against enterotoxigenic Escherichia coli infection. Proc. Natl. Acad. Sci. U.S.A. 110, 11809-11814. doi: 10.1073/pnas.1301975110

Watkins, J. D., Sholukh, A. M., Mukhtar, M. M., Siddappa, N. B., Lakhashe, S. K., Kim, M., et al. (2013). Anti-HIV IgA isotypes: differential virion capture and inhibition of transcytosis are linked to prevention of mucosal R5 SHIV transmission. AIDS 27, F13-F20. doi: 10.1097/QAD.0b013e32836 0eac6

Westerhof, L. B., Wilbers, R. H., van Raaij, D. R., Nguyen, D. L., Goverse, A., Henquet, M. G., et al. (2014). Monomeric IgA can be produced in planta as efficient as IgG, yet receives different N-glycans. Plant Biotechnol. J. 12, 1333-1342. doi: 10.1111/pbi.12251

Westerhof, L. B., Wilbers, R. H., van Raaij, D. R., van Wijk, C. Z., Goverse, A., Bakker, J., et al. (2015). Transient expression of secretory IgA in planta is optimal using a multi-gene vector and may be further enhanced by improving joining chain incorporation. Front. Plant Sci. 6:1200. doi: $10.3389 /$ fpls.2015.01200

Woof, J. M., and Mestecky, J. (2005). Mucosal immunoglobulins. Immunol. Rev. 206, 64-82. doi: 10.1111/j.0105-2896.2005.00290.x

Xiong, E., Li, Y., Min, Q., Cui, C., Liu, J., Hong, R., et al. (2019). MZB1 promotes the secretion of J-chain-containing dimeric $\operatorname{IgA}$ and is critical for the suppression of gut inflammation. Proc. Natl. Acad. Sci. U.S.A. 116, 13480-13489. doi: 10.1073/pnas. 1904204116

Yoo, E. M., Coloma, M. J., Trinh, K. R., Nguyen, T. Q., Vuong, L. U., Morrison, S. L., et al. (1999). Structural requirements for polymeric immunoglobulin assembly and association with J chain. J. Biol. Chem. 274, 33771-33777. doi: $10.1074 / j b c .274 .47 .33771$

Conflict of Interest: The authors declare that the research was conducted in the absence of any commercial or financial relationships that could be construed as a potential conflict of interest.

Copyright (๑) 2020 Göritzer, Goet, Duric, Maresch, Altmann, Obinger and Strasser. This is an open-access article distributed under the terms of the Creative Commons Attribution License (CC BY). The use, distribution or reproduction in other forums is permitted, provided the original author(s) and the copyright owner(s) are credited and that the original publication in this journal is cited, in accordance with accepted academic practice. No use, distribution or reproduction is permitted which does not comply with these terms. 